Estudios Pedagógicos XXXIX, Número Especial 1: 59-74, 2013

INVESTIGACIONES

\title{
Análisis de la disposición pedagógica de los futuros profesores para usar las TIC
}

\author{
Analysis of the pedagogical available of preservices teacher to use ICT
}

Análise da disposição dos futuros professores para o uso das TIC

\author{
Jose Garrido M., ${ }^{\mathrm{a}}$ David Contreras G., ${ }^{\mathrm{b}}$ Christian Miranda J. ${ }^{\mathrm{c}}$ \\ âPontificia Universidad Católica de Valparaíso. Telf.: 322274347. Correo electrónico: jgarrido@ucv.cl \\ bontificia Universidad Católica de Valparaíso. Telf.: 322274707. Correo electrónico: dcontrer@ucv.cl \\ ${ }^{\mathrm{c}}$ Universidad de Chile. Telf.: 229787868. Correo electrónico: christian.miranda@u.uchile.cl
}

\begin{abstract}
RESUMEN
Este artículo busca analizar la disposición para utilizar TIC que manifiestan estudiantes de carrera de pedagogía, a partir del cruce de sus creencias sobre el aporte educativo de dichas tecnologías y sus preferencias sobre lo que deben ser las clases y la manera de usar tecnologías digitales en ellas. El estudio, parte del Proyecto FONDECYT de Iniciación n ${ }^{\circ} 11110455$, utiliza un diseño de investigación de tipo mixto que involucra inicialmente una exploración de datos sobre una muestra-tipo de 12 estudiantes, para luego profundizar el análisis con una muestra nacional de estudiantes $(\mathrm{n}=424)$ proveniente de 13 universidades. Los resultados muestran la existencia de una tipología con disposiciones que pueden transformarse en la base para innovar con tecnologías digitales en la formación de los futuros docentes.
\end{abstract}

Palabras Clave: Formación Inicial Docente, tecnologías digitales, disposiciones.

\begin{abstract}
This article seeks to analyze the willingness to use ICT demonstrated by future teachers from encountering their beliefs on the educational contribution of these technologies and their preferences about how the classes should be, and how to use digital technologies in the classroom. This study, part of the FONDECYT project $\mathrm{N}^{\circ} 11110455$, uses a mixed research design which involves, initially, an exploratory interview on a sample of 12 students and then, a statistical analysis with a national sample of students $(n=424)$ from 13 universities. The results show the existence of a typology with provisions that can become an opportunity to innovate with digital technologies in the future teachers' formation.
\end{abstract}

Keywords: preservice teachers, digital technology, willingness.

\section{RESUMO}

Este artigo procura analisar a disposição de usar TIC dos futuros professores cruzando as suas crenças sobre a contribuição educativa dessas tecnologias e as suas preferências sobre como deveriam ser as aulas com o uso de tecnologias digitais. Este estudo, tem como partida o projeto FONDECYT 11110455, utiliza um método de pesquisa mista, inicialmente, envolve a verificação de dados numa amostra de 12 alunos e, em seguida, uma análise estatística com uma amostra nacional de estudantes $(\mathrm{n}=424)$ de 13 universidades. Os resultados mostram a existência de uma tipologia das disposições que podem ser uma oportunidade para a inovação em tecnologias digitais no ensino dos futuros professores. 
Palavras-chave: professores preservice, tecnologia digital, disponíveis.

\section{INTRODUCCIÓN}

Existe una racionalidad argumentativa arraigada en los sistemas educacionales que atribuye a las tecnologías de información y comunicación (TIC) una capacidad innovadora que puede plasmarse en mejores ambientes y situaciones de aprendizaje, sin embargo, y a pesar de los esfuerzos desplegados para implementar políticas de integración de estas tecnologías en los procesos de enseñanza y aprendizaje, la evidencia empírica muestra la existencia de una brecha entre dicha expectativa y el valor agregado de innovación real que muestran las prácticas formativas (Sancho, 2012). Frente a este dilema, diversos autores (Castro, 2007; Ávalos, 2003) fijan su atención en el papel que le cabe a la formación inicial del profesorado como etapa que debe facilitar la construcción de repertorios prácticos innovadores para una enseñanza para el aprendizaje. En este sentido, las evidencias nacionales e internacionales muestran un bajo nivel de uso de las tecnologías digitales en las aulas de formación inicial de profesores, lo cual constata la carencia experiencial que viven los futuros docentes para aprender a utilizar, como parte de su formación profesional, estas tecnologías (OCDE, 2009). Por contrapartida, los hallazgos muestran que los estudiantes a edades más tempranas y bajo diversas modalidades son usuarios activos de las TIC en sus prácticas habituales

A partir de este contexto de análisis, surge como un punto de interés analizar y caracterizar de qué manera los estudiantes de carreras de pedagogía se posicionan respecto al uso con fines formativos (o pedagógicos) de las tecnologías digitales y qué tipo de disposición manifiestan para realizar (o desarrollar) prácticas innovadoras de enseñanza con ellas. Por lo tanto, lo que este artículo propone es identificar las creencias que poseen los estudiantes sobre lo que implican las TIC en el espacio educativo y analizar su vínculo con las diferentes maneras de usarlas en prácticas de aula propias de sus formación profesional.

Para estudiar este fenómeno se ha implementado, en el marco del Proyecto FONDECYT de Iniciación 11110455, un diseño de investigación de tipo mixto en modalidad explicativosecuencial que implica dos momentos de aplicación de instrumentos. En un primer momento, la aplicación de entrevistas de tipo cualitativa a una muestra-tipo de estudiantes de carreras de pedagogía $(n=12)$ con el fin de caracterizar el tipo de creencias que tienen sobre el papel de las TIC en la educación, particularmente en el ámbito formativo. En un segundo momento, la aplicación de un cuestionario estandarizado a una muestra nacional de estudiantes de pedagogía $(n=424)$ para identificar el papel innovador que atribuyen a las tecnologías digitales en las prácticas de aula. Los resultados muestran diferentes disposiciones para innovar con tecnologías digitales entre quienes aspiran a convertirse en futuros profesores, lo que es discutido en el marco de las oportunidades y barreras del proceso de apropiación de tecnologías digitales en las carreras de pedagogía.

Como ha señalado Litwin, la construcción de una didáctica tecnológica como cuerpo de conocimiento referido a la prácticas de enseñanza para el aprendizaje "necesita incorporar las mejores tradiciones de las propuestas de la tecnología educativa...lograr este propósito hace necesario analizar las tecnologías en los marcos políticos, económicos y culturales actuales" (Litwin, 2005: 18). Considerando las complejidades que tiene la formación inicial de docentes para adecuarse a las demandas que la sociedad está realizando a la educación; y el tránsito hacia 
una didáctica tecnológica para la docencia universitaria, particularmente, se torna relevante para la formación inicial del profesorado analizar la manera en que las TIC están siendo pensadas por aquellos que en el próximo tiempo asumirán el proceso de formación de la nueva generación de estudiantes. Desde esta perspectiva, este artículo contribuye al objetivo de este número especial destinado a la apropiación, problemáticas y desafíos que implica la presencia e incorporación de las tecnologías de información y comunicación en educación.

\section{2. $\quad$ DISCUSIÓN TEÓRICA}

\subsection{TIC COMO PROBLEMÁTICA FORMATIVA}

El uso habitual de las TIC en diversos ámbitos del quehacer humano ha generado cambios en la configuración de las prácticas sociales. En efecto, un mayor número de personas, y a edades más tempranas, interactúan con ambientes digitales para desarrollar diversas actividades individuales y colectivas (ej. acceso a la información). Como resultado, hay nuevas formas de construcción de las relaciones y realizaciones humanas (Goldman et al., 2008; Weber y Mitchell, 2008). Ello está provocando una ruptura de los ejes forjados en la Modernidad para la construcción de la cultura, la organización social y la certidumbre sobre lo que deben ser y hacer profesiones e instituciones (Coll y Monereo, 2008; Bauman, 2006). Esta exposición permanente a las TIC también está cambiando las prácticas de acceso y producción de información, las que se realizan en ambientes hipertextuales modificando la comunicación y el intercambio de las personas (Area et al., 2008; Gros, 2008; Buckingham, 2005).

La evidencia internacional es consistente en señalar que las generaciones más jóvenes de estudiantes son quienes, en mayor cantidad y para tipo de usos cada vez más diversos, utilizan las tecnologías digitales. El Índice Generación Digital de 2004 a 2008, que mide el acceso y uso de las tecnologías digitales realizado por jóvenes en edad escolar en grandes urbes nacionales, muestra que el $96 \%$ de ellos usan internet (IGD, 2008).

Desde la óptica del género, estudios comparados muestran que en Estados Unidos las tecnologías de carácter más masivo (ej. telefonía celular) tienen una tasa homogénea de participación entre hombres y mujeres, no obstante, los usuarios más sofisticados y quienes muestran una mayor dedicación e intensidad en el uso de tecnologías digitales tienden a ser hombres (Horrigan, 2007). Los datos para el caso chileno permiten aseverar que, frente a similares indicadores, la distribución es heterogénea de acuerdo a patrones etarios, socioeconómicos y de género (PNUD, 2006).

Respectos a usos específicos de ambientes digitales, el estudio de Rideout et al. (2005) establecen que, en 2004, las personas de entre 8 y 18 años jugaban en promedio 49 minutos diarios, es decir, las personas en edad escolar dedican un poco más de tiempo a los videojuegos que a leer diarios, revistas o libros (43 minutos), y levemente menos tiempo del que dedican a hacer tareas (50 minutos). En este mismo sentido, Salinas y Sánchez (2009) explicitan que en el contexto rural chileno la experiencia y las expectativas de las comunidades han cambiado con la llegada de Internet, generando una demanda por la integración simbólica y el reconocimiento cultural que la sociedad en su conjunto puede y debe hacer a los sectores rurales gracias a esta herramienta. 
Como parte del debate sobre estos cambios sociales y culturales (Castell, 2005), la inserción de TIC en el ámbito educativo está demandando cambios en las prácticas pedagógicas de los docentes (De Pablos, 2010; Vegas y Peltrow, 2008). Demanda que tiene sus raíces en las transformaciones del contexto histórico, pero también en las formas de trabajar, aprender y pensar de los actuales alumnos que viven en una sociedad medial con procesos de globalización complejos y con tensiones identitarias importantes. En Chile, la estrategia utilizada para abordar esta problemática se ha centrado en democratizar el acceso a las tecnologías digitales en el sistema escolar por medio de la instalación de infraestructura y la alfabetización digital de los docentes (Sánchez y Salinas, 2008; CIDE-IGL-UAH, 2004).

A pesar de estos esfuerzos por posibilitar el acceso, los resultados son desalentadores. En efecto, tomando como referente los resultados de la prueba SIMCE-TIC aplicada en 2010 (Alarcón et al., 2013), solo el 3,64\% de los estudiantes chilenos logra un desempeño "Avanzado" en el uso de TIC, esto es, son capaces de resolver las tareas solicitadas utilizando habilidades tecnológicas y cognitivas de orden superior agrupadas en las dimensiones de información, comunicación, ética e impacto social. La interpretación de estos resultados refleja lo que se ha denominado como segunda brecha digital (Hargittai, 2002; Atewell, 2001; Montgomery, 2000), la cual se vincula a las capacidades concretas de los jóvenes de sacar provecho del computador, lo cual dependería de una combinación de su capital económico, cultural y social (Brunner, 2001). En este contexto la misma prueba SIMCE-TIC consigna (Gráfico 1) que sobre el $57 \%$ de los estudiantes advierte que nunca utiliza las tecnologías digitales en actividades en el aula.

Gráfico 1. Frecuencia de uso de tecnología en la escuela clasificada por nivel de logro

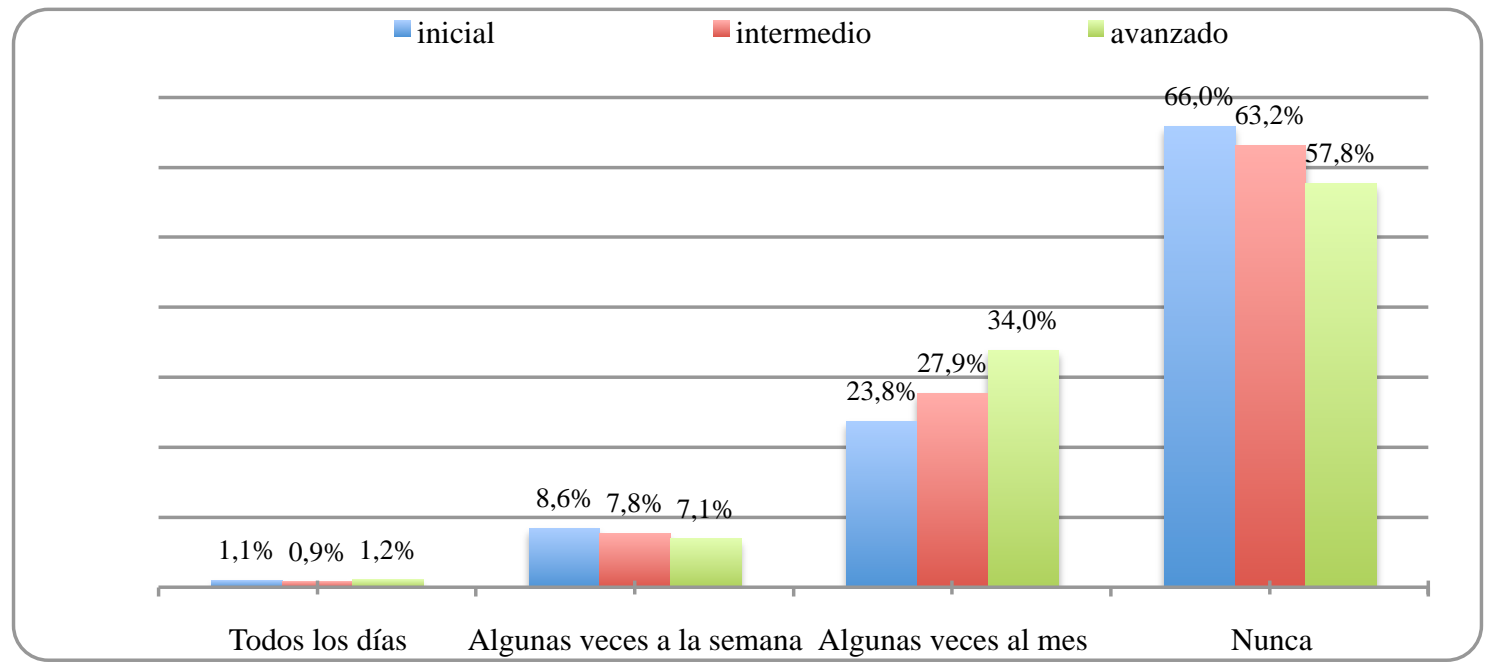

A partir de lo anterior, hoy es vigente la discusión sobre el rol y responsabilidad que tiene el profesorado sobre el desarrollo de las habilidades y competencias que se esperan y son necesarias para que las personas puedan utilizar las TIC. En tal sentido, es la formación inicial de docentes (FID) la que acapara el interés de organismos internacionales y gubernamentales para enfrentar el desafío de incorporar las TIC en el espacio escolar. En efecto, organismos como la OCDE (2009), han impulsado estudios con la intención de conocer qué sucede con el uso de TIC en las carreras de pedagogía. Los resultados obtenidos indican que éstas no son usadas como un 
elemento importante en el desarrollo de los objetivos formativos de dichas carreras. Esto implica que, paradojalmente, a pesar de los requerimientos del campo laboral (ej. escuelas) y de las orientaciones que la política pública impulsa para incorporar estos medios, académicos y estudiantes de pedagogía los usan marginalmente en sus desempeños (OCDE, 2009).

En esta misma línea, un estudio reciente (CET-CEPPE, 2010) confirma que en las carreras de pedagogía existe un proceso incipiente y poco definido de incorporación de las TIC, constatando que éstas aún transitan por una etapa inicial de integración: aceptar y aprender a utilizarlas. De este modo, los estudiantes de pedagogía y los profesores recién egresados experimentan pocas instancias formales para aprehender y aprovechar el potencial innovador de las tecnologías instaladas en los establecimientos educativos del país. En este mismo sentido, Carrasco et al. (2012) evidencian que en las aulas universitarias se desconoce y desaprovecha el tipo y manera en que los estudiantes aprenden fuera de éstas.

\subsection{TIC Y UNA NUEVA GENERACIÓN DE ESTUDIANTES}

Los procesos de globalización han llevado a investigadores a plantear la hipótesis de la emergencia de una nueva generación de estudiantes con atributos socio-cognitivos que los distinguiría de las generaciones anteriores. Estos conformarían lo que algunos denominan "nativos digitales" (Prensky, 2001) o "aprendices del nuevo milenio" (Pedró, 2006): ¿Quiénes son exactamente los aprendices del nuevo milenio? Este es un término ampliamente usado para designar a aquellas generaciones que nacieron a partir de 1980, y que han crecido en un contexto donde la tecnología digital forma una inextricable parte de la cotidianeidad; en otras palabras, aquellos que han sido mediados por la tecnología.

Para Prensky (2001), los "nativos digitales" son quienes han pasado toda su vida usando computadores, videojuegos, reproductores de música digital, cámaras de vídeo, teléfonos celulares y herramientas de la era digital y, al mismo tiempo, han crecido rodeados por ellas. Ellos estarían habituados a recibir información con gran rapidez, gustan de los procesos paralelos y multitareas, prefieren los gráficos antes que los textos, optan por el acceso aleatorio (ej. hipertextos), interactúan mejor cuando están en red, gustan de gratificaciones instantáneas y recompensas constantes y prefieren los juegos antes que el trabajo 'serio'.

La discusión principal se plantea sobre el alcance del desarrollo de habilidades e intereses distintivos respecto a las generaciones anteriores, y la consiguiente influencia de esto sobre la necesidad de una adaptación de los métodos y técnicas de la enseñanza. Por un lado, el mundo extraescolar contrastaría con el mundo de la escuela y, por otro, estos rasgos incrementarían la brecha generacional entre profesores y alumnos. Los primeros, denominados "inmigrantes digitales" -aquellos que no nacieron en el mundo digital pero que, más tarde en algún punto de sus vidas, se convierten en usuarios de diferentes tipos de tecnologías-, tendrían que enfrentarse a aprendices con un lenguaje distinto, destrezas y requerimientos nuevos para los cuales no estarían preparados (Sánchez et al., 2011).

Según Prensky (2001), los instructores inmigrantes digitales hablan un lenguaje desactualizado -el de la era predigital- y luchan para educar a una población que habla un lenguaje completamente nuevo. Otros autores emplean conceptos análogos, tales como: generación red (Oblinger y Oblinger, 2005) o generación gamer (Carstens y Beck, 2005), los que describen la existencia de un conjunto de prácticas sociales que se basan en la incorporación 
de herramientas digitales. Por ejemplo, para la actual generación es cotidiana la comunicación inmediata o las conversaciones mediante el uso de celulares, entre otras.

Las distintas formas a través de las cuales los miembros de esta generación pueden llegar a aprender, comunicar y divertirse constituyen razones que pueden explicar la creciente popularidad de las tecnologías que apuntan a las redes sociales. Precisamente, esta popularidad puede ayudar a entender por qué los jóvenes cada vez dedican mayor tiempo al uso de medios digitales domésticos (ej. video consolas) en desmedro de la televisión (Pedró, 2006). Sin duda, la actividad recurrente con estas tecnologías sugiere algún moldeamiento sobre sus nociones acerca de qué es la comunicación, el conocimiento, el aprendizaje e incluso sus propios valores personales y sociales (Garrido, 2013).

En este marco, Prensky (2001) proyecta que el uso continuo de las tecnologías digitales tendrá implicaciones en el desarrollo de las competencias intelectuales, pudiendo generar estructuras de pensamiento muy distintas a las conocidas. En esta línea, Papert (1994) acuñó el término "grasshopper mind" para designar la tendencia a cambiar rápidamente de un tema a otro, algunas veces hacia delante y hacia atrás, en lugar de prestar atención de forma continua e intensa a un único objeto. De este modo, los jóvenes serían impacientes si la información no la obtienen de manera instantánea y tenderían a no fijar la atención en una misma cosa durante mucho tiempo. Conforme a lo que expresa Pedró (2006), estos estudiantes: i) acceden a la información principalmente a partir de fuentes digitales; ii) dan prioridad a las imágenes en movimiento y a la música por encima del texto; iii) se sienten cómodos realizando tareas múltiples simultáneamente y; iv) obtienen conocimientos procesando información discontinua y no lineal.

Si se sigue la lógica anterior, podría ocurrir que los cambios resultantes en los nuevos aprendices sean distantes o estén en conflicto con las prácticas de enseñanza tradicionales fundadas en la palabra y en la organización secuencial de los saberes.

Asumiendo la existencia de estas diferencias, surge como elemento central de análisis el significado y alcance de lo que es innovar con estas tecnologías en los procesos de enseñanza y aprendizaje. Al respecto, diversos estudios sobre innovación en educación coinciden en señalar que tras este concepto complejo y polisémico se manifiestan componentes explícitos e implícitos de carácter ideológico, cognitivo, ético y afectivos que relevan la influencia de la subjetividad en lo que se define como innovar, la que presenta siempre un principio teleológico de intencionalidad (Carbonell, 2001; Rivas, 2000). Entre los factores que se identifican como causantes de la resistencia a la innovación en educación, se halla el papel que juega la actitud, discurso y acción que evidencian profesores y estudiantes, especialmente por la constatación de que cualquier proceso de cambio se realiza sobre un algo preexistente que se sitúa en la intersección de experiencias y un contexto (Abrami et al., 2004). Sobre este último, se constata la existencia de una disposición socio-afectiva de los docentes y estudiantes a innovar, la que, no obstante, estaría condicionada por factores como las prácticas institucionalizadas y la inseguridad disciplinar, expresadas en una actitud "defensiva" ante estos inconvenientes (Miranda, 2006).

En síntesis, es posible afirmar que para analizar el para qué y el cómo los estudiantes de pedagogía utilizan las tecnologías digitales -herramientas propias de su generación-, resulta necesario indagar en sus creencias y la manera en que éstas fundan su intención y acción. Estos estudiantes, como aprendices digitales, poseen un conjunto de creencias formadas o en transición de formarse que están forjadas por el uso habitual de tecnologías, y desde ellas dialogan con las prácticas que se realizan a lo largo de su formación. Es en la amalgama de estos usos recurrentes, creencias y prácticas educativas con TIC donde esta investigación pone el acento. 


\section{DISEÑO DE INVESTIGACIÓN}

El diseño de investigación utilizado es de tipo mixto, secuencial y explicativo (Creswell, 2009; Teddlie y Tashakkori, 2009). Esto ha significado realizar dos etapas de aplicación de instrumentos. El primero mediante el uso de entrevistas semiestructuradas y estandarizadas, aplicadas con el fin de caracterizar el tipo de creencias que tienen los estudiantes de carreras de pedagogía sobre el papel de las TIC en el proceso formativo. El segundo a través de la elaboración y aplicación de un cuestionario destinado a identificar el nivel de innovación que se atribuye a las tecnologías digitales en las prácticas de aula.

a. Etapa 1. Utilizando un muestreo de perfil tipo, se seleccionó a un grupo de 12 estudiantes de carreras de pedagogía de la Pontificia Universidad Católica de Valparaíso, sobre la base de los siguientes criterios:

-Carrera de pedagogía cursada: estudiantes de pedagogía en Educación Primaria, Educación Especial y Educación Infantil.

-Trayectoria como estudiante de pedagogía: estudiantes de primer y último año.

-Género de los estudiantes: hombres y mujeres.

El método utilizado para aproximarse a las creencias sobre el papel de las TIC en los procesos formativos fue la aplicación de un ciclo de dos entrevistas semiestructuradas en modalidad inicio-contrastación. El análisis de sus resultados se realizó utilizando los principios propuestos por la fenomenografía (Marton y Yan Pong, 2005; Pang, 2003). El primero de ellos, denominado aspectos referenciales de la experiencia, consideró una lectura inductiva de las entrevistas agrupadas según el muestreo-tipo utilizado. Las entrevistas fueron analizadas de acuerdo a un análisis del discurso que incluyó los métodos de Actos del Habla, Pragmática y Polaridades. De esta manera, se obtuvo como resultado 29 categorías descriptivas.

El segundo principio, denominado aspecto estructural de la experiencia, implicó la comparación constante de las 29 categorías descriptivas con el propósito de identificar las similitudes y variaciones de significados y sentidos que surgen entre ellas. Finalmente, estos significados y sentidos se han agrupado de acuerdo a un tópico explicativo, por ejemplo, aquellos relacionados con el aprendizaje o aquellos relacionados con aspectos sociales. Sus resultados permitieron elaborar cinco "Metacategorías Descriptivas" que representan las creencias que los estudiantes de carreras de pedagogía tienen sobre lo que es el fenómeno de las TIC y su papel en la formación.

b. Etapa 2. Se aplicó un cuestionario destinado a identificar el nivel de innovación atribuido a las TIC en los procesos de formación. Éste incluyó la recopilación de información sobre la frecuencia y el nivel de uso de las tecnologías digitales declarada por los estudiantes, las creencias sobre lo que aportan dichas tecnologías a la educación, y la intencionalidad pedagógica-tecnológica para usarlas en situaciones formativas. Se incorporaron las cinco metacategorías descriptivas sobre el fenómeno de las TIC obtenidas en la etapa anterior y se vincularon con ítems relacionados con métodos pedagógicos y la integración tecnológica de las TIC adaptados a partir de la matriz de habilidades de enseñanza y competencias tecnológicas propuestas para medir la apropiación de las TIC en contextos formativos (Mei-Chuen, Wang y Lin, 2012). 
La aplicación del instrumento se realizó a una muestra nacional de 424 estudiantes de la carrera de pedagogía en Educación Básica pertenecientes a 13 universidades chilenas, tal como se resume en la tabla 1 :

Tabla 1. Distribución de estudiantes para cuestionario

\begin{tabular}{|lccc|}
\hline \multicolumn{1}{c}{ Institución } & F & M & Total \\
\hline Pontificia U. Católica de Valparaíso & 23 & 1 & 24 \\
\hline U. de los Lagos & 7 & 1 & 8 \\
\hline U. Autónoma de Chile & 32 & 7 & 39 \\
\hline U. Bolivariana & 17 & 8 & 25 \\
\hline U. del Bío-Bío & 17 & 9 & 26 \\
\hline U. Católica de la Santísima Concepción & 75 & 11 & 86 \\
\hline U. Católica de Temuco & 16 & 3 & 19 \\
\hline U. de Atacama & 25 & 5 & 30 \\
\hline U. de La República & 6 & 3 & 9 \\
\hline U. Playa Ancha de Ciencias de la Educación & 49 & 5 & 54 \\
\hline U. Pedro de Valdivia & 29 & 3 & 32 \\
\hline U. Santo Tomas & 17 & 2 & 19 \\
\hline U. de Tarapacá & 39 & 13 & 52 \\
\hline Total & $\mathbf{3 5 2}$ & $\mathbf{7 1}$ & $\mathbf{4 2 3}$ \\
\hline
\end{tabular}

\section{4. $\quad$ ANÁLISIS DE RESULTADOS}

Un primer tipo de análisis permite evidenciar la existencia de cinco creencias principales que poseen los estudiantes de carreras de pedagogía sobre lo que caracteriza a las tecnologías digitales en los procesos de formación (ver Tabla 1). Estas muestran que los informantes reconocen que aquellas poseen el potencial para transformar los procesos formativos, sin embargo, ello no logra plasmarse por una ausencia de iniciativa de los docentes formadores al momento de diseñar las clases, situación que impide la existencia de experiencias innovadoras de uso de TIC. No obstante, surge como creencia el hecho de que más allá del bajo uso de tecnologías en las aulas universitarias, éstas sí tienen un papel en las interacciones que se producen entre estudiantes fuera de las aulas de clases.

Un segundo aspecto que puede destacarse es la valoración que se hace del entorno familiar y formativo como inductores del uso de TIC, especialmente por haberse transformado en un medio que otorga un tipo de reconocimiento social entre pares, por haberse naturalizado como una necesidad entre sus familias, y por formar parte de la cultura digital, al menos desde el punto de vista de la gestión, en las propias instituciones de educación superior de la cual forman parte.

Tabla 2. Creencias sobre el papel de las TIC en la Formación.

\begin{tabular}{|c|c|}
\hline Creencia & ción \\
\hline $\begin{array}{l}\text { "Las TIC son una Motivación } \\
\text { para Aprender". }\end{array}$ & $\begin{array}{l}\text { Las TIC generan motivación debido a que permiten trabajar en } \\
\text { entornos basado en lenguajes iconográfico-visuales y porque } \\
\text { favorecen en trabajo autónomo de los propios estudiantes. }\end{array}$ \\
\hline
\end{tabular}




\begin{tabular}{|c|c|}
\hline $\begin{array}{l}\text { "Las TIC No Resultan } \\
\text { Innovadoras dentro del Aula". }\end{array}$ & $\begin{array}{l}\text { A pesar, del creciente uso de tecnologías digitales que se observa al } \\
\text { interior de las carreras universitarias de pedagogía, dichas prácticas no } \\
\text { logran ser innovadoras. Esta situación es producto de la falta de } \\
\text { iniciativa de los docentes y de la ausencia de experiencias } \\
\text { ejemplificadoras para los propios estudiantes. }\end{array}$ \\
\hline $\begin{array}{l}\text { "Las TIC influyen en las } \\
\text { Interacciones fuera del Aula". }\end{array}$ & $\begin{array}{l}\text { Influencia que tienen las tecnologías de información y comunicación } \\
\text { en las actividades que se producen fuera del aula y que se relacionan } \\
\text { con la realización de actividades y comunicación para realizar tareas o } \\
\text { actividades relacionadas con los procesos de formación. Lo que se } \\
\text { manifiesta en las posibilidades para acceder, recuperar e intercambiar } \\
\text { información en modalidades que rompen con maneras espacio- } \\
\text { temporales tradicionales, permitiendo a los estudiantes organizar de } \\
\text { otra manera el tiempo que destina a las actividades fuera del aula. }\end{array}$ \\
\hline $\begin{array}{l}\text { "Las Tecnologías Digitales se } \\
\text { Usan por Presión del } \\
\text { Entorno". }\end{array}$ & $\begin{array}{l}\text { Las tecnologías de información y comunicación se han transformado } \\
\text { en un medio que despierta interés por aspectos tales como el } \\
\text { reconocimiento social que implica poseerlas, su naturalización como } \\
\text { necesidad en las familias y por los requerimientos administrativos o de } \\
\text { gestión de los propios escenarios formativos. }\end{array}$ \\
\hline $\begin{array}{l}\text { "Las TIC pueden Disminuir la } \\
\text { Brecha Social". }\end{array}$ & $\begin{array}{l}\text { TIC poseen un carácter de mediador en la construcción de una nueva } \\
\text { culturización de la sociedad contemporánea. Ofreciendo posibilidades } \\
\text { reales a los sectores más vulnerables de acceder a nuevas } \\
\text { oportunidades. }\end{array}$ \\
\hline
\end{tabular}

Al contrastar estas creencias con la muestra nacional obtenida mediante el cuestionario, se observa (Tabla 2) que para la totalidad de las creencias medidas existe un porcentaje por sobre el $55 \%$ de acuerdo con ellas (se cree totalmente o en gran parte con ellas). De acuerdo a los datos obtenidos, esto permite afirmar que las creencias o convicciones más fuertes que poseen los estudiantes de carreras de pedagogía sobre el papel de las TIC en la realidad educativa actual son: (i) el contexto socioeducativo impulsa su uso habitual en diversas prácticas, aunque muchas veces esto no se justifica $(69,0 \%)$; (ii) la interacción con estas tecnologías están configurando un nuevo tipo de aprendiz que es diferente al de generaciones precedentes (67,6\%); (iii) las tecnologías digitales tienen el potencial de favorecer el aprendizaje a través de su influencia motivadora (67,6\%); y (iv) el estudiantado de las carreras de pedagogía no está preparado para hacer un uso pedagógico de las TIC (67,0\%). A pesar de que el nivel de rechazo o duda manifestado hacia las creencias medidas no supera, para ningún caso, el $50 \%$ de adhesión, igualmente resulta interesante observar que aquellas que son menos compartidas por los estudiantes de pedagogía a nivel nacional apuntan al conocimiento y capacidad para enseñar y aprender usos innovadores de las tecnologías digitales $(45,0 \%)$, y a cuestionar el papel que le caben a estos ambientes como forjadoras de la sociedad actual $(42,1 \%)$.

Tabla 3. Distribución porcentual de nivel de creencias sobre el papel de las TIC

\begin{tabular}{|c|c|c|c|c|}
\hline Creencias & $\begin{array}{c}\text { "Creo } \\
\text { totalmente" }\end{array}$ & $\begin{array}{c}\text { "Creo en } \\
\text { gran parte" }\end{array}$ & $\begin{array}{c}\text { "Creo solo } \\
\text { algunos } \\
\text { aspectos" }\end{array}$ & $\begin{array}{c}\text { No creo } \\
\text { esto" }\end{array}$ \\
\hline TICs motivan y favorecen el aprendizaje. & 29,3 & 38,4 & 26,4 & 5,9 \\
\hline
\end{tabular}




\begin{tabular}{|c|c|c|c|c|}
\hline $\begin{array}{r}\text { TICs configuran un nuevo perfil de } \\
\text { Aprendiz. }\end{array}$ & 28,9 & 38,7 & 27,2 & 5,3 \\
\hline TICs reconfiguran la Comunicación. & 31,3 & 33,7 & 27,4 & 7,6 \\
\hline $\begin{array}{r}\text { TICs se usan aunque no siempre se } \\
\text { necesitan. }\end{array}$ & 30,5 & 38,5 & 22,1 & 8,8 \\
\hline $\begin{array}{r}\text { TICs reconfiguran las Prácticas del } \\
\text { Profesor. }\end{array}$ & 26,4 & 40,1 & 26,3 & 7,1 \\
\hline $\begin{array}{r}\text { TICs determinan lo que es la sociedad } \\
\text { actual. }\end{array}$ & 24,5 & 33,3 & 28,4 & 13,7 \\
\hline $\begin{array}{r}\text { Profesoradolestudiantado de Pedagogía no } \\
\text { está preparado para Innovar ni para } \\
\text { enseñar/aprender con TICs. }\end{array}$ & 23,3 & 31,7 & 25,4 & 19,6 \\
\hline $\begin{array}{r}\text { Estudiantes de Pedagogía no está preparado } \\
\text { para hacer uso de las TICs. }\end{array}$ & 26,2 & 40,8 & 24,2 & 8,8 \\
\hline
\end{tabular}

A partir de esto, se puede afirmar que para los estudiantes de carreras de pedagogía, las tecnologías digitales solo adquieren un valor cualitativo si son capaces de romper con la inercia que provoca la tradición y comodidad relativa que ofrecen las prácticas habituales, situación que solo se puede producir en las mismas prácticas de clases en la universidad, es decir, al mostrar concretamente de qué manera las tareas y actividades de enseñanza que habitualmente se realizan sin el uso de tecnologías digitales, pueden verse enriquecidas al usarlas.

Un segundo tipo de análisis permite caracterizar que el 88,7\% de los estudiantes hace uso de las TIC en sus clases (Tabla 3), existiendo un 69,5\% que señala usarlas siempre o la mayoría de las veces. Esto resulta revelador considerando que a nivel escolar, como ya se ha señalado, un $57 \%$ señala que nunca las usa en sus clases. Este dato muestra una frecuencia e intencionalidad de uso destinada a los procesos formativos.

Tabla 4. Porcentaje de Uso de TIC en Clases

\begin{tabular}{|l|r|}
\hline Frecuencia de Uso & Porcentaje \\
\hline Nunca & 0,2 \\
\hline Algunas Veces & 29,2 \\
\hline La Mayoría de las Veces & 48,3 \\
\hline Siempre & 21,2 \\
\hline
\end{tabular}

Al ahondar en los tipos de uso de TIC dentro de las actividades académicas (Gráfico 2), podemos observar que los estudiantes señalan que el mayor uso semanal de herramientas y ambientes digitales se produce para la elaboración de presentaciones $(70,3 \%)$ y la comunicación con el profesor fuera del horario de clases $(65,1 \%)$. Mientras el menor uso semanal se relaciona con la utilización de Internet para buscar información académica y para intercambiar recursos de aprendizaje. 
Gráfico 2. Frecuencia Porcentual de Uso semanal de TIC para Actividades Formativas

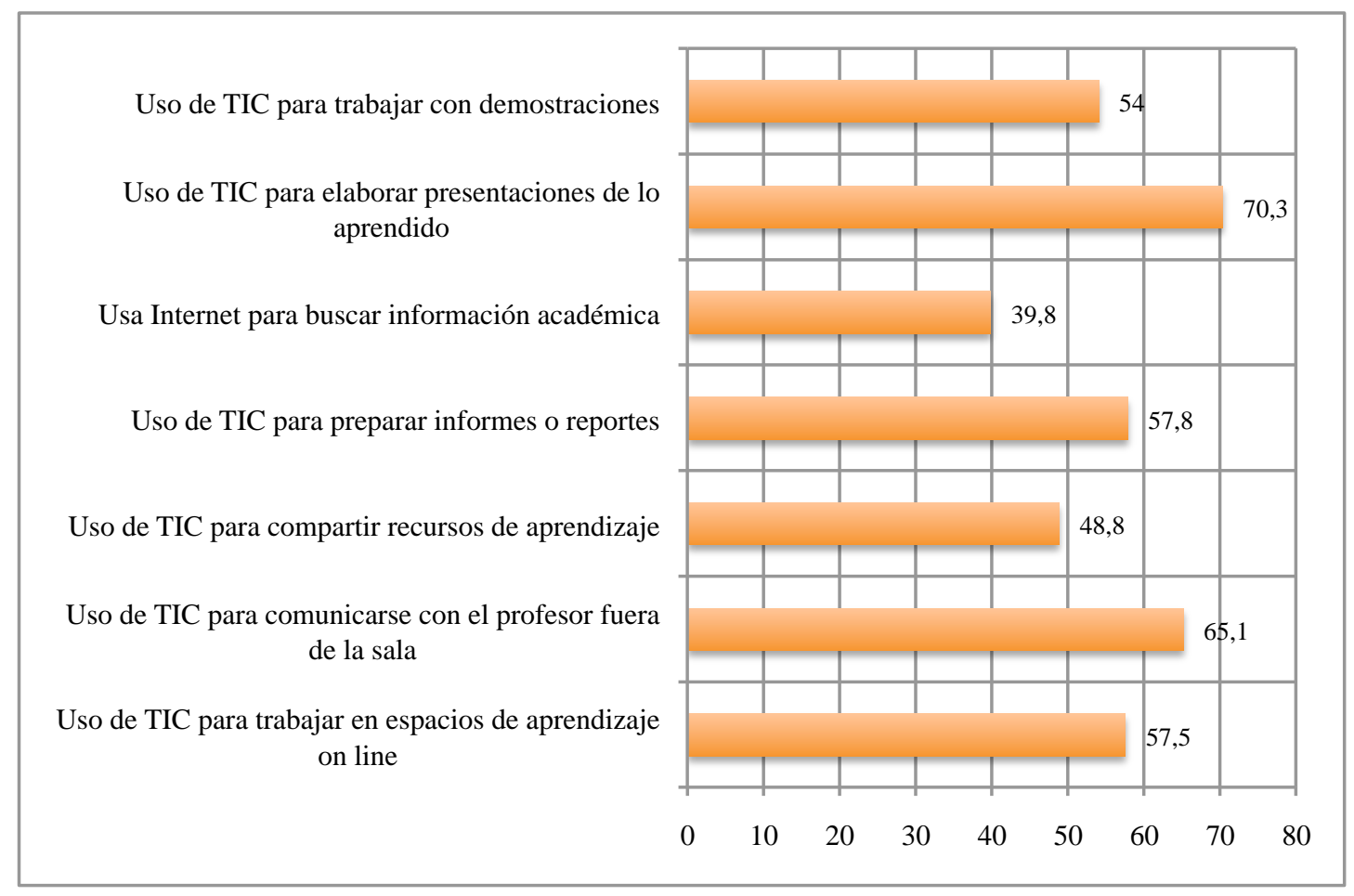

El tercer tipo de análisis realizado es el cruce de la variable "tipo de clase preferida por el estudiante", que considera cuatro descripciones que se alinean con diseños de enseñanza directiva, aprendizaje activo, aprendizaje constructivo y aprendizaje social; con la variable "tipo esperado de uso de TIC en clases", que considera 8 descripciones de incorporación de las tecnologías. Ambas variables fueron tomadas del modelo dimensional de integración de TIC propuestos por Mei-Chuen, Wang y Lin (2012) y adaptadas al contexto nacional de formación inicial de docentes para su incorporación en el cuestionario utilizado (Garrido y Ponce, 2012). Al cruzar ambas respuestas (Tabla 4) es posible apreciar la existencia de algunas tendencias y disposiciones que manifiestan los estudiantes de carrera de pedagogía para utilizar las TIC en procesos de formación.

Tabla 5. Distribución Porcentual de la Disposición Tipo de Enseñanza/Tipo de Uso de TIC de los Estudiantes de FID.

\begin{tabular}{|c|c|c|c|c|c|}
\hline & $\begin{array}{c}\text { Enseñanza } \\
\text { Directiva }\end{array}$ & $\begin{array}{c}\text { Aprendizaje } \\
\text { Activo }\end{array}$ & $\begin{array}{c}\text { Aprendizaje } \\
\text { Constructivo }\end{array}$ & $\begin{array}{l}\text { Aprendizaje } \\
\text { Social }\end{array}$ & Total \\
\hline $\begin{array}{l}\text { No tengo mayor Interés o no tengo el } \\
\text { Conocimiento necesario para incorporar } \\
\text { TICs en mis labores como estudiante de } \\
\text { pedagogía. }\end{array}$ & 0,22 & 0,45 & 6,01 & 0,22 & 6,90 \\
\hline $\begin{array}{l}\text { Uso TICs solo en pequeñas actividades } \\
\text { que me ayuden a completar las diferentes } \\
\text { tareas que me solicitan. }\end{array}$ & 1,11 & 1,56 & 1,11 & 1,56 & 5,35 \\
\hline
\end{tabular}




$\begin{aligned} & \text { Uso TICs principalmente para observar y } \\ & \text { tomar nota de presentaciones en power } \\ & \text { point preparadas por profesor. }\end{aligned}$
$\begin{aligned} & \text { Uso diferentes herramientas de } \\ & \text { comunicación información ofrecidas por }\end{aligned}$
$\begin{aligned} & \text { Internet antes, durante y después de las } \\ & \text { clases para ayudarme a profundizar mis } \\ & \text { aprendizajes. }\end{aligned}$

Se puede observar que a nivel de los procesos de enseñanza los estudiantes de pedagogía prefieren diseños donde ellos asuman roles de mayor protagonismo. Así, por ejemplo, el 33,85\% muestra preferencia por clases que utilicen actividades en las cuales tengan que debatir $\mathrm{y}$ profundizar la información y la construcción de conocimiento con otros estudiantes y personas más allá de los límites del aula; el 28,29\% manifiesta preferir clases con actividades en las cuales se les solicite realizar tareas de representación de lo aprendido, por ejemplo, mediante mapas conceptuales; finalmente, el $25,17 \%$ lo hace por actividades formativas que impliquen resolver situaciones problemáticas o desarrollar proyectos de investigación. Solo el 12,69\% manifiesta su gusto por clases basadas en la exposición del profesor, algunas lecturas claves y evaluaciones al final de cada unidad para demostrar lo aprendido.

La forma en que estas preferencias se distribuyen respecto a diferentes maneras de utilizar TIC en actividades formativas, muestra que la mayor tendencia $(37,19 \%)$ se observa en el uso de diferentes herramientas de comunicación e información ofrecidas por Internet para buscar, ampliar y profundizar los temas de aprendizaje vistos en clases. Esto es consistente con la otra preferencia de uso de tecnologías digitales expuesta por los estudiantes: la utilización de herramientas para trabajar de manera autónoma y a un ritmo propio los aprendizajes esperados en las diferentes asignaturas.

La clara preeminencia de estas dos maneras de utilizar las tecnologías digitales y su transversalidad entre las diferentes formas de organizar la enseñanza, permiten afirmar que los estudiantes de carrera de pedagogía ponen en evidencia su disposición a utilizar estas tecnologías en la medida que se propicie oportunidades para un trabajo autónomo que se adecue a su propios avances de aprendizaje, situación que implica intencionar el uso de TIC más allá del tiempo y espacio tradicional establecido por el aula. 
Es importante señalar que estos resultados deben ser leídos considerando las limitaciones propias del estudio y sus oportunidades de profundización, por ejemplo, la necesidad de contrastar estos hallazgos con las disposiciones e intencionalidades que manifiestan los docentesformadores con el fin de establecer la influencia y relaciones de unas sobre otras. Así como equilibrar y ampliar manera proporcional la muestra de informantes desde las universidades estudiadas, con el fin mejorar la diversidad de informantes.

\section{CONCLUSIONES}

A pesar de la presencia extendida de las tecnologías de información y comunicación en el sistema escolar, sus aportes al mejoramiento de las prácticas de aula son episódicas y siguen representando más bien una promesa que una realidad (Savage y McGoun, 2012; Bates, 2009; OCDE, 2009). El cúmulo de evidencias que muestran su potencialidad para desarrollar aprendizajes y para enriquecer los escenarios formativos (Jones, 2012; Martínez, 2011; Bartolomé et al., 2010; Area et al., 2008) exige repensar la manera de impulsar la apropiación pedagógica con un sentido curricular innovador y en el papel que le cabe en ello a la formación inicial de docentes.

Los estudios muestran que en este nivel formativo el uso de TIC es escaso y con un bajo nivel de innovación (CET-CEPPE, 2010). Desde un principio eco-sistémico de la realidad educativa, es decir, desde la interpretación de los significados, intenciones y maneras utilizadas por los actores para dar sentido y uso a las tecnologías digitales, develar las disposiciones que subyacen a ello resulta esencial desde un punto de vista comprensivo de la realidad educativa.

En esta investigación se ha podido establecer que los estudiantes de carrera de pedagogía tienden a creer que las tecnologías digitales son, efectivamente, una oportunidad para innovar en las prácticas de aula, situación que ellos asocian al surgimiento de un nuevo perfil de estudiante, el que es usuario habitual de estas tecnologías. Sin embargo, también creen que el aporte de aquéllas no logra plasmarse en las aulas universitarias, principalmente porque ni ellos ni el profesorado que los forma poseen los conocimientos ni están preparados para esto. Un dato que permite contextualizar esta creencia es que los propios estudiantes informan que los principales usos que hacen con las TIC durante sus actividades formativas se vinculan con la elaboración de presentaciones y la comunicación con el docente, es decir, principalmente actividades centradas en la enseñanza.

Al analizar las disposiciones que manifiestan los estudiantes para utilizar las tecnologías digitales, se puede apreciar que de manera diferente a lo que sucede en sus clases, los estudiantes aspiran a un uso que priorice la autonomía y ritmo de avance frente al aprendizaje, así como vincular dicho uso a una mirada que rompe con el eje espacio-temporal del aula. Estas disposiciones apuntan en la dirección de resituar el uso de TIC desde una mirada de transformación de las prácticas habituales realizadas por los docentes. En esta línea, es interesante recordar que algunos autores han argumentado la existencia de una escala progresiva de cuatro tipos de uso de las TIC en educación, que va desde de la adquisición de habilidades tecnológicas como un fin en sí mismo hasta el proceso de integración estructural de la tecnología que reforma la estructura de funcionamiento de una institución educacional. El nivel previo al cambio estructural se relaciona con la integración y papel transformador de las habilidades 
tecnológicas de los estudiantes en un proceso de cambio que afecta tanto la forma en que ocurre el aprendizaje, como la definición es aprender (Fluck, 2010; Downes, 2002).

Los resultados obtenidos permiten establecer la existencia de algunas disposiciones para utilizar las TIC que son manifestadas por los estudiantes de pedagogía en su rol como aprendices en general, y como aprendices digitales en particular ¿De qué manera la autonomía del proceder y la deslocalización del espacio de enseñanza dan cuentan de una nueva manera de aproximarse al conocimiento y el proceso de aprendizaje? ¿de qué manera estas disposiciones representan una manera de innovar en el proceso formativo mediado por TIC? y ¿de qué manera estas disposiciones surgen producto de las prácticas sociales y habituales con tecnologías digitales que se realizan fuera del contexto formativo? estas son interrogantes que deben ser abordadas desde la investigación, especialmente porque en espacios no formativos las innovaciones y cambios mediados por TIC son asumidos por los estudiantes mediante experiencias y vivencias directas (Goldman et al., 2008) que no son aprehendidas por los escenarios formativos reglados.

Esto queda reflejado en el bajo desarrollo de experiencias, procedimientos y repertorios de enseñanza innovadores con los que egresan los estudiantes de pedagogía, lo cual debilita sus opciones para desenvolverse en escenarios escolares reales minimizando y desnudando la debilidad del aporte formativo atribuible a la FID (Knowles, 2004; Rivas, 2000), en este caso, para desarrollar las promesas y expectativas que se espera de la apropiación con sentido formativo de las tecnologías de información y comunicación.

\section{REFERENCIAS BIBLIOGRÁFICAS}

Abrami, P., Poulsen, C. y Chambers, B. (2004). Teacher motivation to implement an educational innovation: factors differentiating users and non-users of cooperative learning. Educational Psychology, vol.24, n.2, 201-216.

Alarcón, P., Álvarez, X., Hernández, D. y Maldonado, D. (2013). SIMCE TIC: Diseño, aplicación y resultados. Una evaluación de siglo XXI para las habilidades TIC de los estudiantes chilenos. En Ceppe-País Digital-Enlaces (Eds.), Desarrollo de habilidades digitales para el siglo XXI en Chile ¿Qué dice el SIMCE TIC? (pp. 15-40). Santiago: LOM.

Area, M., Gros, B. y Marzal, M. (2008). Alfabetizaciones y tecnologías de la información y la comunicación. Madrid: Síntesis.

Attewell, P. (2001). The first and second digital divides. Sociology of Education, vol.74, n.3, 252-259.

Ávalos, B. (2003). La formación docente inicial en Chile. Report Digital Observatory for Higher Education in Latin America and the Caribbean. Caracas: IESALC-UNESCO.

Bartolomé, A., Cano, E. y Compaño, P. (2011). Los Blogs como instrumentos de evaluación de competencias. En T. Pagés, A. Cornet y J. Pardo (Eds.), Buenas prácticas docentes en la universidad. Modelos y experiencias en la Universidad de Barcelona (pp. 77-85). Barcelona: Octaedro/ICE-UB.

Bates, A. (2009). ¿Se comprende realmente lo que es el E-Learning? En A. Gewerc (Ed.), Políticas, prácticas e investigación en tecnología educativa (pp. 109-132). Barcelona: Octaedro/ICE-UB.

Bauman, Z. (2006). Vida Líquida. Barcelona: Paidós.

Brunner, J. (2001). Modernidad: Centro y periferia. Claves de lectura. Estudios Públicos, n.83, 1-15.

Buckingham, D. (2005). Educación en medios. Alfabetización, aprendizaje y cultura contemporánea. Barcelona: Paidós.

Carbonell, J. (2001). El profesorado y la innovación educativa. En P. Cañal del León (Ed.), La innovación educativa (pp. 11-26). Madrid: Universidad Internacional de Andalucía-Akal. 
Carrasco, R., Jadue, F., Letelier, M. y Oliva, C. (2012). Estudio exploratorio sobre aprendizaje no formal e informal de estudiantes y egresados universitarios. Calidad de la Educación, n.36, 150-183.

Carstens, A. y Beck, J. (2005). Get ready for the gamer generation. TechTrends: Linking Research \& Practice to Improve Learning, vol.49, n.3, 22-25.

Castells, M. (2005). Globalización, desarrollo y democracia: Chile en el contexto mundial. Santiago: Fondo de Cultura Económica.

Castro, A. (2007). Formación inicial y profesión docente. En J. Brunner y C. Peña (Eds.), La reforma al sistema escolar: Aportes para el debate (pp. 103-120). Santiago: Universidad Diego Portales.

CET-CEPPE. (2010). Tecnologías de la información y de las comunicaciones en la formación inicial docente. Reporte nacional del estudio TIC-FID 2009. Santiago: IIE-UFRO.

CIDE-IGL-UAH. (2004). Evaluación en profundidad programa red tecnológica educacional enlaces: Informe final. Santiago: Ministerio de Educación.

Coll, C. y Monereo, C. (2008). Educación y aprendizaje en el siglo XXI: Nuevas herramientas, nuevos escenarios, nuevas finalidades. En C. Coll y C. Monereo (Eds.), Psicología de la educación virtual (pp. 19-53). Madrid: Morata.

Creswell, J. (2009). Research desing, qualitative, quantitative, and mixed methods approaches. Los Ángeles: Sage.

De Pablos, J. (2010). Políticas educativas y la integración de las TIC, a través de buenas prácticas docentes. En J. de Pablos, M. Area, J. Valverde y J. Correa (Eds.), Políticas educativas y buenas prácticas con TIC (pp. 21-41). Barcelona: Graó.

Downes, T. (2002). Blending play, practice and performance: children's use of computer at home. Journal of Educational Enquiry, vol.3, n.2, 21-34.

Fluck, A. (2010). From integration to transformation. En A. Mcdougall, J. Murnane, A. Jones \& N. Reynolds (Eds.), Researching it in education. Theory, practice and future directions (pp. 62-71). New York: Routledge.

Garrido, J. (2013). Videojuegos de estrategia: Algunos principios para la enseñanza. Revista Electrónica de Investigación Educativa, vol. 15, n.1, 62-74.

Garrido, J. y Ponce, C. (2012). Inventory of ICT beliefs in initial teacher training: Toll for identify trends of innovation. Proceedings of ICERI 2012 Conference (pp. 3104-3112), 19th-21st November. Madrid.

Goldman, S., Booker, A. y Mcdermott, M. (2008). Mixing the digital, social, and cultural: Learning, identity and agency in youth participation. In D. Buckingham (Ed.), Youth, identity, and digital media (pp. 185-206). Cambridge: MIT.

Gros, B. (2008). Aprendizajes, conexiones y artefactos: La producción colaborativa del conocimiento. Barcelona: Gedisa.

Hargittai, E. (2002). Second-level digital divide: Differences in people's online skills. Recuperado el 07 de Octubre de 2013, de http://www.eszter.com/research/pubs/hargittai-secondleveldd.pdf

Horrigan, J. (2007). A typology of information and communication technology users. Washington: Pew Internet \& American Life Project.

IGD. (2008). Estudio Índice Generación Digital. VTR, Educarchile y Adimark. Santiago. Chile.

Jones, C. (2012). Networked learnign, stepping beyond the net generation and digital natives. En Disckinck-Holmfeld, L., Hodgson, V. \& McConnell, D. (eds), Exploring the theory, Pedagogy and practice of networked learning (pp. 27-41). New York-Dordrecht-Heidelber-London: Springer.

Knowles, J. (2004). Modelos para la comprensión de las biografías del profesorado en formación y en sus primeros años de docencia. In I. Goodson (Ed.), Historias de vida del profesorado (pp. 149-205). Barcelona: Octaedro.

Litwin, E. (2005). La tecnología educativa en el debate didáctico contemporáneo. En E. Litwin (Ed.), Tecnologías educativas en tiempos de internet (pp. 13-34). Buenos Aires: Amorrortu. 
Martínez, M. (2011). Modelos de enseñanza y aprendizaje con TIC. En M. Cebrián de la Serna y M.J. Gallego (Eds.), Procesos educativos con TIC en la sociedad del conocimiento (pp. 179-189). Madrid: Pirámide.

Marton, F. y Yan Pong, W. (2005). On the unit of description in phenomenography. Higher education research \& development, vol.24, n.4, 335-348.

Mei-Chuen, J., Wang, P. y Lin, I. (2012). Pedagogy technology: A two-dimensional model for teachers' ICT integration. British Journal of Educational Technology, vol.43, n.1, 97-108.

Miranda, C. (2006). Impacto de la formación permanente de profesores sobre sus competencias profesionales. Santiago: Pontificia Universidad Católica de Chile.

Montgomery, K. (2000). Children's media culture in the new milleniun: Mapping the digital landscape. Children and Computer Technology, vol.10, n.2, 145-167.

Oblinger, D. y Oblinger, J. (2005). Educating the net generation. Washington: Educase.

OCDE. (2009). ICT and initial teacher training. Paris: CERI.

Pang, M. F. (2003). Two faces of variation: On continuity in the phenomenographic movement. Scandinavian Journal of Educational Research, vol.47, n.2, 145-156.

Papert, S. (1994). The children's machine: Rethinking school in the age of the computer. New York: Basic Books.

Pedró, F. (2006). The new millennium learners: Challenging our views on ICT and learning: Paris. OECD-CERI.

PNUD. (2006). Desarrollo Humano en Chile. Las nuevas tecnologías: ¿un salto al futuro? Santiago: UNESCO.

Prensky, M. (2001). Digital natives, digital immigrants. On the Horizon, vol.9, n.5, 1-6.

Rideout, V., Roberts, D. y Foehr, U. (2005). Generation m: Media in the lives of 8-18 year-olds. Menlo Park, CA: Henry Kayser Family Foundation.

Rivas, M. (2000). Innovación Educativa. Teoría, procesos y estrategias. Madrid: Síntesis.

Salinas, A. y Sánchez, J. (2009). Digital inclusion in Chile: Internet in rural schools. International Journal of Educational Development, vol.29, n.6, 573-582.

Sánchez, J. y Salinas, A. (2008). ICT \& learning in chilean schools: Lessons learned. Computers \& Education, vol.51, n.4, 1621-1633.

Sánchez, J. y Salinas, A., Contreras, D. y Meyer, E. (2011). Does the New Digital Generation of Learners Exist? A Qualitative Study. British Journal of Education Technology, vol.42, n.4, 543-556.

Sancho, J. (2012). Las muchas decisiones y pasos de un proyecto. In J. Sancho y C. Alonso (Eds.), $L a$ fugacidad de las políticas, la inercia de las prácticas. La educación y las tecnologías de la información y comunicación (pp. 13-20). Barcelona: Octaedro.

Savage, J. y McGoun, C. (2012). Technology, culture and communication. London-New York: Routledge.

Teddlie, C. y Tashakkori, A. (2009). Foundations of mixed methods research: Integrating quantitative and Qualitative approaches in the social and behavioral sciences. Los Angeles-London-New Delhi: Sage.

Vegas, E. y Petrow, J. (2008). Learning in latinoamerica. The challenge for the 21st century. Washington, DC: World Bank.

Weber, S. y Mitchell, C. (2008). Imaging, keyboarding, and posting identities: Young people and new media technologies. In D. Buckingham (Ed.), Youth, identity, and digital media (pp. 25-46). Cambridge: MIT. 